\title{
Microbial diversity and volatile profile of traditional fermented yak milk
}

\author{
Yang Jiang, ${ }^{1}$ Nan Li, ${ }^{2}$ Qi Wang, ${ }^{1,3,4}$ Zhenmin Liu, ${ }^{2 *}$ Yuan-Kun Lee, ${ }^{5}$ Xiaoming Liu, ${ }^{1,3,6 *}$ (b) Jianxin Zhao, ${ }^{1,3,6}$ \\ Hao Zhang, ${ }^{1,3,4}$ and Wei Chen ${ }^{1,3,4,7}$ \\ ${ }^{1}$ State Key Laboratory of Food Science and Technology, Jiangnan University, Wuxi 214122, China \\ ${ }^{2}$ State Key Laboratory of Dairy Biotechnology, Shanghai Engineering Research Center of Dairy Biotechnology, Dairy Research Institute, \\ Bright Dairy and Food Co. Ltd., Shanghai 200436, China \\ ${ }^{3}$ School of Food Science and Technology, Jiangnan University, Wuxi 214122, China \\ ${ }^{4}$ National Engineering Research Centre for Functional Food, Wuxi 214122, China \\ ${ }^{5}$ Department of Microbiology and Immunology, Yong Loo Lin School of Medicine, National University of Singapore, Singapore 117597 \\ ${ }^{6}$ International Joint Research Laboratory for Probiotics at Jiangnan University, Wuxi 214122, China \\ ${ }^{7}$ Beijing Innovation Centre of Food Nutrition and Human Health, Beijing Technology and Business University, Beijing 100048, China
}

\begin{abstract}
Previous research reported that fermented yak milk had a diverse microbial composition. For this study, raw yak milk, qula, and fermented yak milk samples were collected from the Aba Tibetan autonomous region of China. The genus and species microbial composition of these samples were analyzed by high-throughput sequencing of $16 \mathrm{~S}$ rRNA and groEL gene amplicons, and the volatile profile of the samples was quantified by gas chromatography-mass spectrometry. The results indicated variation in abundance of microbiota at the genus level among the fermented yak milk samples, with Lactobacillus as the most abundant genus in the majority of samples, ranging from 41.6 to $98.3 \%$. The volatile profile of the samples varied among those collected from different villages. Correlations between bacterial composition and volatile compounds of the samples were also observed. Lactobacillus displayed a significant correlation with volatile compounds such as benzaldehyde, 2,3-pentanedione, ethanol, and ethyl acetate, whereas the samples with relatively high abundance of Streptococcus and Lactococcus displayed relatively low contents of volatile compounds.
\end{abstract}

Key words: fermented yak milk, microbial diversity, volatile profile

\section{INTRODUCTION}

The yak (Bos grunniens) is a long-haired bovid found throughout the Himalaya region of southern Central Asia, the Tibetan Plateau, Mongolia, and Russia, with over $95 \%$ of the world's total yak population currently

Received April 5, 2019.

Accepted September 10, 2019.

*Corresponding authors: liuzhenmin@brightdairy.com and liuxm@ jiangnan.edu.cn in China (Zhang et al., 2008). Fermented yak milk is a traditional dairy product in northwestern China that is generally prepared using qula - a traditional product made by defatting, acidifying, and air-drying yak milk (Duan et al., 2008; Yang et al., 2018) and fermenting at 4 to $15^{\circ} \mathrm{C}$ for 12 to $36 \mathrm{~h}$ - as the starter culture.

The diverse microbial composition of fermented yak milk has been reported previously (Bai et al., 2010; Bao et al., 2012; Liu et al., 2012; Liu et al., 2015). At the genus level, Firmicutes are dominated by Lactobacillus, Streptococcus, Lactococcus, and Leuconostoc, whereas Ascomycota were mainly Saccharomycodes. At the species level, Lactobacillus species, including Lactobacillus delbrueckii ssp. bulgaricus, Lactobacillus helveticus, Lactobacillus fermentum, and Lactobacillus plantarum were detected, with the presence of Streptococcus thermophilus and Lactococcus lactis in certain samples as well. Although revealing, previous research mainly focused on identification of the microbiota composition; further analysis beyond the relative abundance of individual genera is desired.

Although the 16S rRNA profiling methods have been widely used to investigate microbial flora at phylum and genus level, its limitation at species level has been reported, and genetic markers such as the groEL gene have been developed for identification of lactic acid bacteria in various samples (Bergonzelli et al., 2006; Blaiotta et al., 2008; Shevtsov et al., 2011; Hu et al., 2017; Milani et al., 2018). In previous studies we have developed effective approaches for the analysis of $B i$ fidobacterium and Lactobacillus in fecal and naturally fermented samples using the groEL gene (Hu et al., 2017; Xie et al., 2019). Thus, a more detailed analysis of microbial diversity of traditionally fermented yak milk samples would provide useful information for screening and application of lactic acid bacteria.

In addition, few studies have investigated the volatile profile of traditional fermented yak milk (Chi et al., 
Table 1. Sampling information for different yak milk products from 15 families in 5 villages in Ruoergai, the Aba Tibetan autonomous region of China ${ }^{1}$

\begin{tabular}{|c|c|c|c|c|}
\hline $\begin{array}{l}\text { Family } \\
\text { code }\end{array}$ & $\begin{array}{l}\text { Raw yak } \\
\text { milk (M) }\end{array}$ & Qula (Q) & $\begin{array}{l}\text { Fermented } \\
\text { yak milk }(\mathrm{T})\end{array}$ & $\begin{array}{l}\text { Sampling } \\
\text { village }\end{array}$ \\
\hline A1 & MA1 & QA1 & TA1 & Dagenggou \\
\hline $\mathrm{A} 2$ & MA2 & QA2 & TA2 & Dagenggou \\
\hline A3 & - & QA3 & - & Dagenggou \\
\hline $\mathrm{A} 4$ & MA4 & QA4 & TA4 & Dagenggou \\
\hline B1 & MB1 & QB1 & TB1 & Axi \\
\hline $\mathrm{B} 2$ & - & QB2 & - & Axi \\
\hline $\mathrm{C} 1$ & - & $\mathrm{QC} 1$ & TC1 & Duoma \\
\hline $\mathrm{C} 2$ & $\mathrm{MC} 2$ & $\mathrm{QC} 2$ & TC2 & Duoma \\
\hline D1 & - & QD1 & TD1 & Banyou \\
\hline D2 & MD2 & QD2 & TD2 & Banyou \\
\hline D3 & MD3 & QD3 & TD3 & Banyou \\
\hline D4 & MD4 & QD4 & TD4 & Banyou \\
\hline D5 & - & QD5 & TD5 & Banyou \\
\hline E1 & - & QE1 & TE1 & Xiangdong \\
\hline $\mathrm{E} 2$ & - & QE2 & TE2 & Xiangdong \\
\hline
\end{tabular}

${ }^{1}$ Fermented yak milk was made from raw yak milk, with qula as the starter culture, by individual families.

2017). Therefore, in the present study, the objective was to evaluate the microbial diversity of yak milk, qula, and fermented yak milk from Ruoergai in the Aba Tibetan autonomous region of China. Furthermore, the variation in abundance at genus level of lactic acid bacteria and at species level of Lactobacillus among the fermented yak milk samples were evaluated, as well as the volatile profile of the fermented yak milk products.

\section{MATERIALS AND METHODS}

\section{Sampling Region}

Samples were collected from Ruoergai, the Aba Tibetan autonomous region of China, located at $32^{\circ} 56^{\prime}$ to $34^{\circ} 19^{\prime} \mathrm{N}$ and $102^{\circ} 08^{\prime}$ to $103^{\circ} 39^{\prime} \mathrm{E}$. It has a subarctic climate, with an elevation of approximately $3,500 \mathrm{~m}$ above sea level, average precipitation of $656.8 \mathrm{~mm}$ per year, and annual average temperature of $1.1^{\circ} \mathrm{C}$. During the sampling period, the temperature ranged from 4 to $15^{\circ} \mathrm{C}$.

\section{Sampling}

A total of 36 samples, including 8 yak milk, 15 qula, and 13 fermented yak milk, were collected from 15 families across 5 villages of Ruoergai, the Aba Tibetan autonomous region of China, including Dagenggou, Axi, Douma, Banyou, and Xiangdong, from June 5 to 10, 2018. Information about the samples is shown in Table 1. Samples with the same sampling code are from the same family, and fermented yak milk was made by individual families from raw yak milk with qula as the starter culture. During the sampling, 30 to $50 \mathrm{~mL}$ of raw milk and fermented milk, and around $30 \mathrm{~g}$ of qula were collected in triplicate from each household, packaged in
50-mL sterile plastic bottles, and frozen immediately. After sampling the samples were transferred on ice to the laboratory within $24 \mathrm{~h}$.

\section{Physical and Chemical Analysis}

Proximate Composition. The total solids content was determined by drying $5 \mathrm{~g}$ of each sample at $110^{\circ} \mathrm{C}$ for $7 \mathrm{~h}$ in a capsule according to AOAC method 990.20. The determination of fat contents of the samples was carried out gravimetrically, according to AOAC method 2000.18, using petroleum ether as solvent for Soxhlet extraction. The protein content was measured using a Kjeltec 8400 (Foss Tecator, Hoganas, Sweden) according to AOAC method 991.23. Lactose was determined according to AOAC method 896.01.

$p H$ Determination and Titratable Acidity. Samples' pH was measured using a digital $320 \mathrm{pH}$ meter (Mettler Toledo, Zurich, Switzerland). Ten grams of each sample was diluted with $70 \mathrm{~mL}$ of distilled water and mixed thoroughly for $\mathrm{pH}$ measurement. The acidity of the sample was determined by titration, following the method described by the AOAC (947.05) using phenolphthalein (Riedel-de Haën AG, Seelze, Germany) as an indicator.

\section{High-Throughput Sequencing of 16S rRNA Amplicons}

Total bacterial DNA was extracted from each sample using the FastDNA SPIN kit for soil and the Fast-Prep Instrument (Qbiogene Inc., Carlsbad, CA) according to the manufacturer's instructions. The extracted DNA was examined on agarose gel and quantified using the Quant-iTTM HS ds-DNA assay kit (Invitrogen, Paisley, 
UK) in combination with the QuBitTM fluorometer. The bacterial V3-V4 16S rRNA region was amplified with the primer pairs $341 \mathrm{~F}$ (5'-CCTAYGGGRBGCASCAG-3') and 806R (5'-GGACTACNNGGGTATCTAAT- $\left.3^{\prime}\right)$. The amplification procedure were performed according to previous research (Jia et al., 2016). The groEL gene of the Lactobacillus genus was amplified with the primer pairs 308F (5'-GCYGGTGCWAACCCNGTTGG-3') and 806R (5'-AANGTNCCVCGVATCTTGTT-3'), and the amplification procedure was conducted according to previous protocols developed in our laboratory (Hu et al., 2017). The PCR products were multiplexed in a single pool in equimolar amounts, based on the QuBit quantification data. The PCR product pool was then purified using the solid-phase reverse immobilization method of the Quant-iT PicoGreen dsDNA assay kit (Qiagen, Hilden, Germany) and sequenced at Fasteris SA (Geneva, Switzerland). The TurSeq DNA LT sample preparation kit (Illumina Inc., San Diego, CA) was used for amplicon library preparation, and the sequencing reaction was performed using a MiSeq Illumina instrument (Illumina Inc.) with V3 chemistry, generating 300-bp paired-end reads. The bioinformatic sequence analysis was conducted as described by Mao et al. (2015).

\section{Volatile Compounds Analysis}

The volatile compounds of fermented yak milk were detected and quantified by headspace solid-phase micro-extraction GC/MS. Six milliliters of each sample was kept in 20-mL clear glass screw top vials at room temperature for $10 \mathrm{~min}$ before analysis. Each sample was added with decanoic acid ethyl ester $(0.05 \mathrm{mg} / \mathrm{mL}$; Sigma-Aldrich, Milwaukee, WI) as an internal standard. The selection of DVB/CAR/PDMS fiber (divinylbenzene/carboxen/polydimethylsiloxane; Supelco, Bellefonte, PA), column and GC program were based on the references, with modification (Hettinga et al., 2008; Settachaimongkon et al., 2014; Dan et al., 2017). After equilibration of the samples for $30 \mathrm{~min}$ at $50^{\circ} \mathrm{C}$, the fiber was exposed to the headspace for $5 \mathrm{~min}$ at $50^{\circ} \mathrm{C}$. A Trace $1300 \mathrm{GC}$ fitted with a single-quadrupole ISQ MS (Thermo Fisher Scientific Inc., Waltham, MA,) and a Rtx-Wax capillary column (30 m, 0.25-mm internal diameter, $0.25-\mathrm{mm}$ thickness). Desorption was performed within $2 \mathrm{~min}$ in splitless mode at $240^{\circ} \mathrm{C}$. The injection temperature was $240^{\circ} \mathrm{C}$, and the transfer line and ion source were at $240^{\circ} \mathrm{C}$. The mass detector was operated in electron impact mode at a voltage of 70 $\mathrm{eV}$, and mass spectra were recorded with a mass range of 33 to $500 \mathrm{~m} / z$ with 0.2 scans/s. Helium was used as the carrier gas at a constant linear velocity of 15.0 $\mathrm{mL} / \mathrm{min}$. The temperature program was the following: $30^{\circ} \mathrm{C}$ (3 min), from 30 to $225^{\circ} \mathrm{C}$ at $15^{\circ} \mathrm{C} / \mathrm{min}$, held for 5 min at $225^{\circ} \mathrm{C}$. The NIST 2011 standard mass spectral database was used to identify volatile compounds based on retention time and mass-spectral similarity match (more than 85\%). Authentic standards were applied to confirm the marker volatile compounds of the assignments. All analysis was conducted in triplicate.

\section{Statistical Analysis}

Data on the compositions of yak milk and fermented yak milk samples were analyzed for statistical significance via ANOVA using the statistical package SPSS 21.0 (SPSS Inc., Chicago, IL). Principal component analysis, partial least squares discrimination analysis, and hierarchical clustering analysis were performed using SIMCA 14.1 (Umetrics AB, Umea, Sweden). Differences between samples were analyzed using Tukey's test and considered to be significant when $P<0.05$.

\section{RESULTS AND DISCUSSION}

\section{Proximate Composition, pH, and Titratable Acidity}

The proximate composition (total solids, fat, protein, lactose), $\mathrm{pH}$, and titratable acidity of yak milk (Supplemental Table S1; https://doi.org/10.3168/jds.2019 -16753) were similar to the results of previous studies of yak milk (He et al., 2011). The mean total solids content, protein, lactose content, and fat content of the yak milk were $15.10 \pm 0.90 \%(\mathrm{SD}), 4.12 \pm 0.54 \%, 4.71$ $\pm 0.83 \%$, and $5.69 \pm 0.88 \%$, respectively. The mean titratable acidity of yak milk was $23.04 \pm 1.31^{\circ} \mathrm{T}$, and the $\mathrm{pH}$ value was in the range of 6.36 to 6.62 .

Variation in the composition of fermented yak milk samples was observed (Supplemental Table S2; https: //doi.org/10.3168/jds.2019-16753) and expected for naturally fermented dairy products considering factors such as raw milk, starter culture, and fermentation conditions. The mean total solids, fat, and lactose contents of the fermented yak milk samples were $14.78 \pm 1.47 \%$, $5.25 \pm 1.02 \%$, and $2.13 \pm 0.71 \%$, close to the compositions reported in a previous study of naturally fermented yak milk (Zhang et al., 2008). Protein content and titratable acidity of our samples were $2.80 \pm 0.31 \%$ and $107.40 \pm 29.09^{\circ} \mathrm{T}$, lower than previously reported values of $5.37 \%$ and $158^{\circ} \mathrm{T}$ (Zhang et al., 2008).

\section{Microbial Diversity Analysis}

The Simpson_1-D, Chao-1, and Shannon_H indices are shown in Table 2; these indices are used to characterize the diversity and richness of each sample. For the 3 types of collected samples, the highest levels of 
diversity and richness were observed in yak milk (mean Simpson_1-D $=0.73$, mean Chao-1 $=600.5)$, followed by qula (mean Simpson_1-D = 0.49, mean Chao-1 = 362.6) and fermented yak milk (mean Simpson_1-D $=0.32$, mean Chao- $1=238.6$ ), which illustrated that the diversity and richness of microbiota in the samples decreased significantly during fermentation. The high microbial diversity and richness of yak milk was probably due to the milking and exposure of the raw milk to the environment before fermentation. As a starter culture, qula was usually air-dried after fermentation; the decrease of $\mathrm{pH}$ value and water activity might partly inhibit excessive microbial growth, as reported in fermented meat products (Fontana et al., 2016). For fermented yak milk, preparation, including heating of raw yak milk and addition of qula as starter culture, and overnight fermentation all contributed to the lower diversity and richness compared with raw milk and qula, as also observed previously by Bao et al. (2012).

As shown in the heat map, Firmicutes was the most abundant phylum (Supplemental Figure S1A; https:/ /doi.org/10.3168/jds.2019-16753), and Lactobacillus, Lactococcus, and Streptococcus were the most abundant genera in the samples (Supplemental Figure S1B; https://doi.org/10.3168/jds.2019-16753). Firmicutes dominated in all of the samples, and similar results have been observed in naturally fermented foods, including fermented dairy products and fermented llama meat sausages (Hunt et al., 1997), probably due to the ability of lactic acid bacteria to produce acid and antibacterial substances, which help them to outcompete other microbial populations during fermentation.

At the genus level, Pearson correlation analysis was performed on the bacterial composition (Figure 1). The correlation coefficients of the genus compositions of yak milk and qula ranged from -0.2 to 0.9 (with a mean value of 0.26 ), whereas the coefficients of the genus compositions of qula and fermented yak milk were in the range 0.3 to 0.82 (with a mean value of 0.56 ). The results indicated that the genus compositions of qula and fermented yak milk were more similar than the genus compositions of yak milk and fermented yak milk, which might be attributed to the fact that qula is typically added as the starter culture during preparation of fermented yak milk.

\section{Bacterial Composition of Fermented Yak Milk}

The relative bacterial abundances at phylum, genus, and Lactobacillus species levels of fermented yak milk are shown in Figure 2. The taxonomical assignment showed that $87 \%$ of the sequences were classified at phylum level, $92 \%$ at genus level, and $99 \%$ at Lacto-
Table 2. $\alpha$-Diversity values of lactic acid bacteria in samples of yak milk, qula, and fermented yak milk from Ruoergai, the Aba Tibetan autonomous region of China

\begin{tabular}{|c|c|c|c|}
\hline $\begin{array}{l}\text { Sampling } \\
\text { code }^{1}\end{array}$ & Simpson_1-D & Shannon_H & Chao-1 \\
\hline MA1 & 0.91 & 3.61 & 541.50 \\
\hline MA2 & 0.62 & 1.77 & 245.40 \\
\hline MA4 & 0.53 & 1.37 & 545.50 \\
\hline MB1 & 0.30 & 0.86 & 184.40 \\
\hline $\mathrm{MC} 2$ & 0.88 & 3.37 & $1,060.00$ \\
\hline MD2 & 0.81 & 2.33 & 500.50 \\
\hline MD3 & 0.93 & 3.54 & 753.50 \\
\hline MD5 & 0.84 & 3.31 & 973.00 \\
\hline QA1 & 0.71 & 1.69 & 363.00 \\
\hline QA2 & 0.43 & 1.16 & 416.30 \\
\hline QA4 & 0.61 & 1.27 & 331.10 \\
\hline QB2 & 0.68 & 1.71 & 393.20 \\
\hline QC1 & 0.15 & 0.48 & 452.40 \\
\hline $\mathrm{QC} 2$ & 0.56 & 1.25 & 222.50 \\
\hline QD1 & 0.44 & 0.99 & 241.20 \\
\hline QD2 & 0.50 & 1.27 & 349.40 \\
\hline QD4 & 0.57 & 1.26 & 354.90 \\
\hline QD5 & 0.54 & 1.32 & 251.00 \\
\hline QE1 & 0.22 & 0.62 & 614.00 \\
\hline TA1 & 0.80 & 2.50 & 568.00 \\
\hline TA2 & 0.48 & 0.87 & 106.00 \\
\hline TA3 & 0.48 & 0.90 & 221.10 \\
\hline TA4 & 0.30 & 0.62 & 329.10 \\
\hline TB1 & 0.16 & 0.39 & 335.30 \\
\hline TB2 & 0.11 & 0.30 & 201.50 \\
\hline TC1 & 0.69 & 1.48 & 306.60 \\
\hline TC2 & 0.26 & 0.59 & 192.60 \\
\hline TD1 & 0.18 & 0.51 & 110.60 \\
\hline TD2 & 0.70 & 1.58 & 430.90 \\
\hline TD3 & 0.39 & 0.69 & 70.67 \\
\hline TD4 & 0.04 & 0.15 & 133.00 \\
\hline TD5 & 0.05 & 0.17 & 73.00 \\
\hline TE1 & 0.07 & 0.20 & 249.10 \\
\hline TE2 & 0.13 & 0.33 & 251.70 \\
\hline
\end{tabular}

${ }^{1}$ Each sampling code comprises an indicator of the type of product ( $\mathrm{M}$ = raw yak milk, $\mathrm{Q}=$ qula, $\mathrm{T}=$ fermented yak milk) and an individual family code. Detailed information on the sampling codes is shown in Table 1 .

bacillus species level. At phylum level, Proteobacteria, Firmicutes, and Bacteroidetes displayed relatively high abundance (Figure 2A), with the abundance of the other phyla, such as Actinobacteria, Tenericutes, and Verrucomicrobia, lower than $0.06 \%$ (data not shown). Firmicutes accounted for more than $99 \%$ at phylum level in 13 of the 15 fermented yak milk samples (TA2, TA3, TA4, TB1, TB2, TC1, TC2, TD1, TD3, TD4, TD5, TE1, and TE2), with $87.67 \%$ in TA1 and $91.35 \%$ in TD2. A similar trend of dominant Firmicutes was observed in previous studies on fermented yak milk (Zhang et al., 2008; Chen et al., 2009).

At genus level (Figure 2B), Lactobacillus was observed as the most abundant genus in 9 samples: TA1 (41.60\%), TA2 $(68.67 \%)$, TA3 $(70.74 \%)$, TA4 $(84.66 \%)$, TC1 (67.57\%), TC2 (94.57\%), TD1 (94.38\%), TD3 $(74.44 \%)$, and TD5 (98.26\%). Streptococcus predominated in 4 samples, TB1 (91.21\%), TD4 (98.09\%), 
TE1 (96.57\%), and TE2 (92.99\%), with Lactococcus predominating in TB2 (94.43\%) and TD2 (46.75\%). We also recorded 16 other genera, such as Acetobacter, Bifidobacterium, and Aggregatibacter, with abundances lower than $0.8 \%$ (data not shown).

The results of the bacterial community at the Lactobacillus species level are shown in Figure 2C. The Lactobacillus species L. delbrueckii was the most abundant in TA1 (99.39\%), TB1 (98.74\%), TD3 (99.38\%), TE1 (99.08\%), and TE2 (98.56\%), whereas L. helveticus was the most abundant species in TA4 (99.33\%), TC1 (99.67\%), TC2 (96.05\%), and TD2 (83.38\%). The results illustrated that generally only one Lactobacillus species was dominant in each sample of the collected fermented yak milk. Besides $L$. delbrueckii and L. helveticus, 5 other Lactobacillus species, including Lactobacillus acidophilus, Lactobacillus casei, L. fermentum, L. plantarum, and Lactobacillus amylovorus were detected with abundances lower than $0.02 \%$ (data not shown).

\section{Volatile Compound Analysis}

Fourteen volatile compounds were identified in the fermented yak milk samples, including 6 acids, 4 ketones, 1 aldehyde, 1 alcohol, 1 ester, and 1 ether (Table 3). Compared with commercial fermented bovine milk samples (data not shown), the fermented yak milk samples generally displayed higher dimethyl disulfide and ethanol content, probably due to differences in microbial composition and fermentation conditions. We should also point out that the volatile profile is highly dependent on the selection of GC column and analytical conditions. In the present study a polar column was chosen, based on the fact that fermented yak milk has high fat content; a more nonpolar column will result in different volatile profile, with more nonpolar volatile compounds.

Principal component analysis of the volatile compounds is shown in Figure 3A, and the first 2 compo-

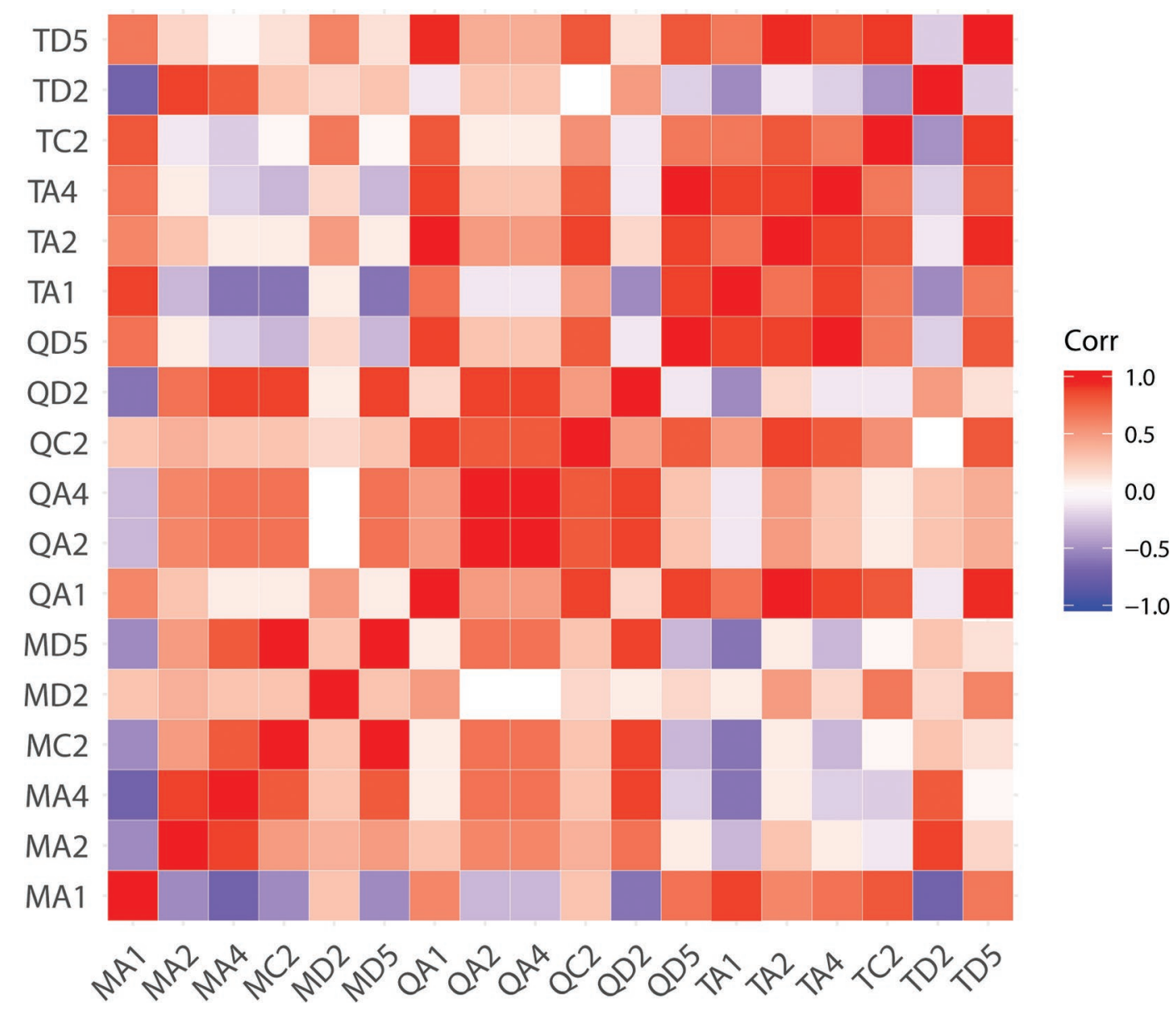

Figure 1. Intercorrelation analysis of bacterial diversity of raw yak milk, qula, and fermented yak milk samples. Pearson coefficient was calculated, and correlation coefficients (Corr) are shown in different colors. Each sampling code (left and bottom edges of the figure) comprises an indicator of the type of product $(\mathrm{M}=$ raw yak milk, $\mathrm{Q}=$ qula, $\mathrm{T}=$ fermented yak milk) and an individual family code. Detailed information on the sampling codes is shown in Table 1. 
nents contributed to $63.7 \%$ of the variation. Samples from villages $\mathrm{C}, \mathrm{A}, \mathrm{B}, \mathrm{D}$, and $\mathrm{E}$ are distributed on the horizontal axis from right to left in the figure. The content of acetic acid in the samples collected from village C ranged from 98.08 to $177.27 \mu \mathrm{g} / \mathrm{kg}$, significantly higher than those of villages D (7.85 to $91.3 \mu \mathrm{g} / \mathrm{kg}$ )

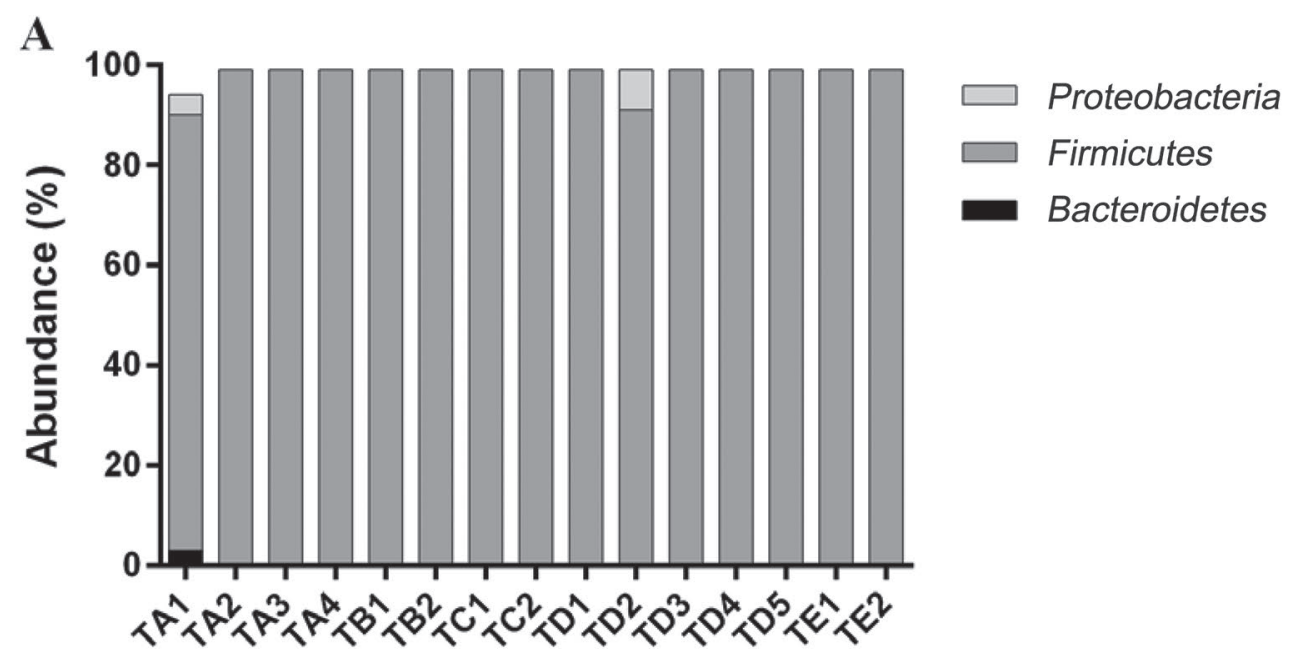

B
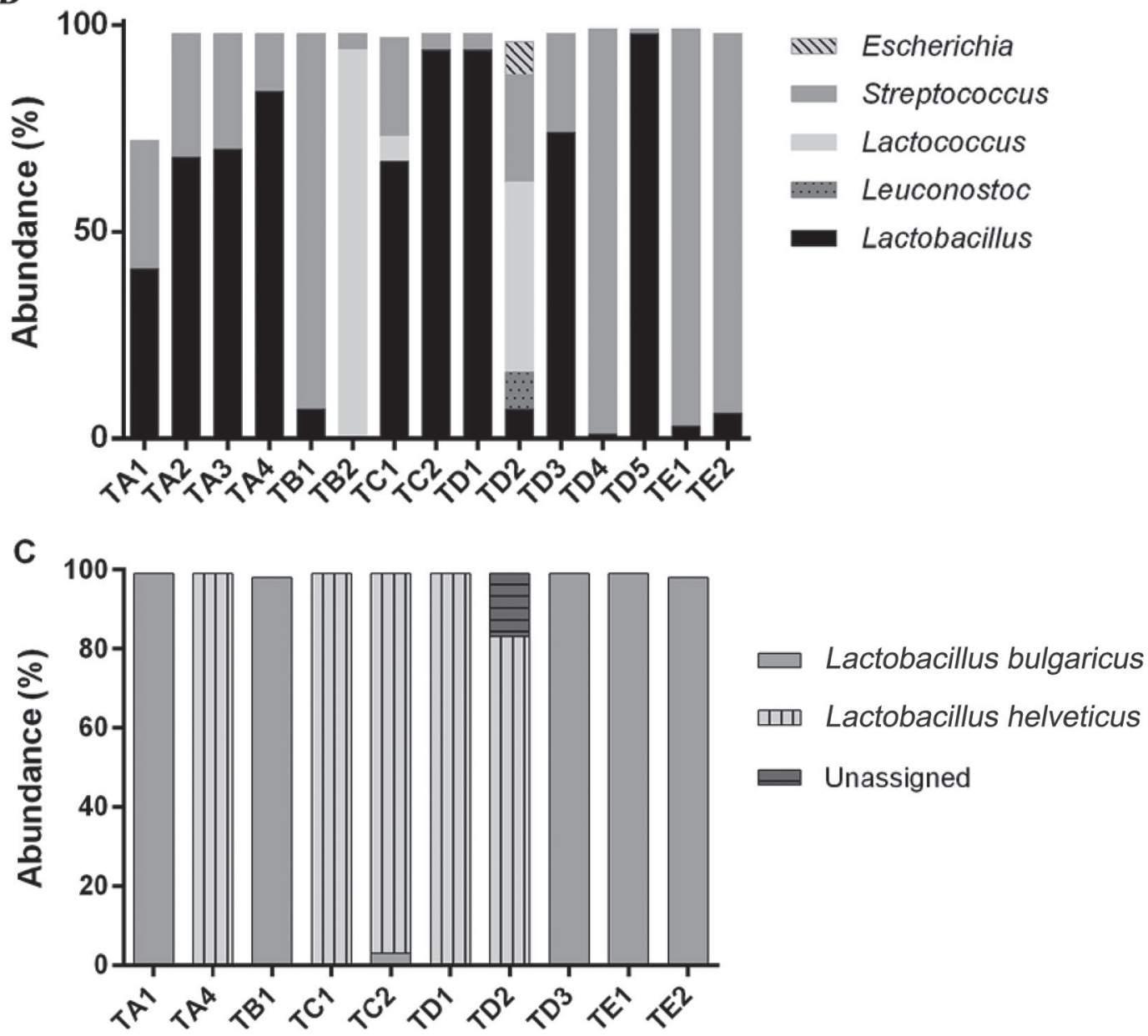

Figure 2. Relative abundance of bacteria in fermented yak milk (A) at phylum level, (B) at genus level (relative abundance $>1 \%$ ), and (C) at species level within the genus Lactobacillus. Each sampling code (bottom edge of each panel) comprises an indicator of the type of product (M $=$ raw yak milk, $\mathrm{Q}=$ qula, $\mathrm{T}$ = fermented yak milk) and an individual family code. Detailed information on the sampling codes is shown in Table 1. 


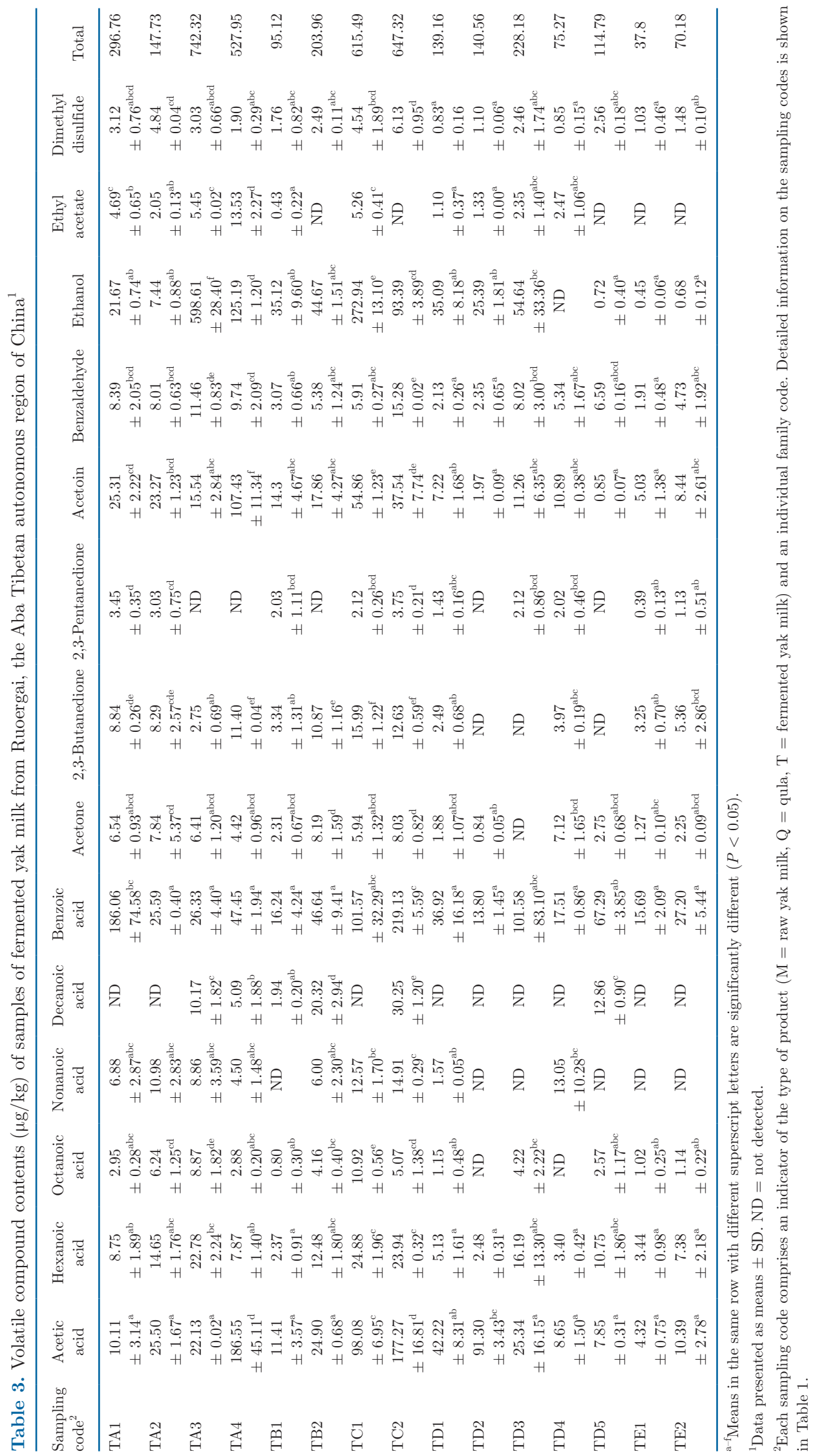


A

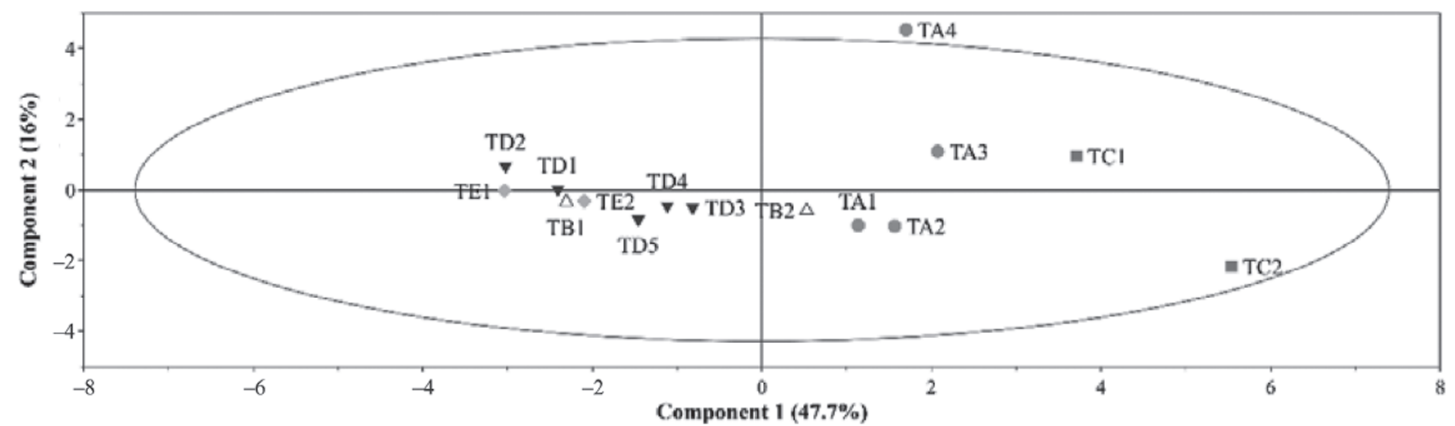

$\mathrm{B}$

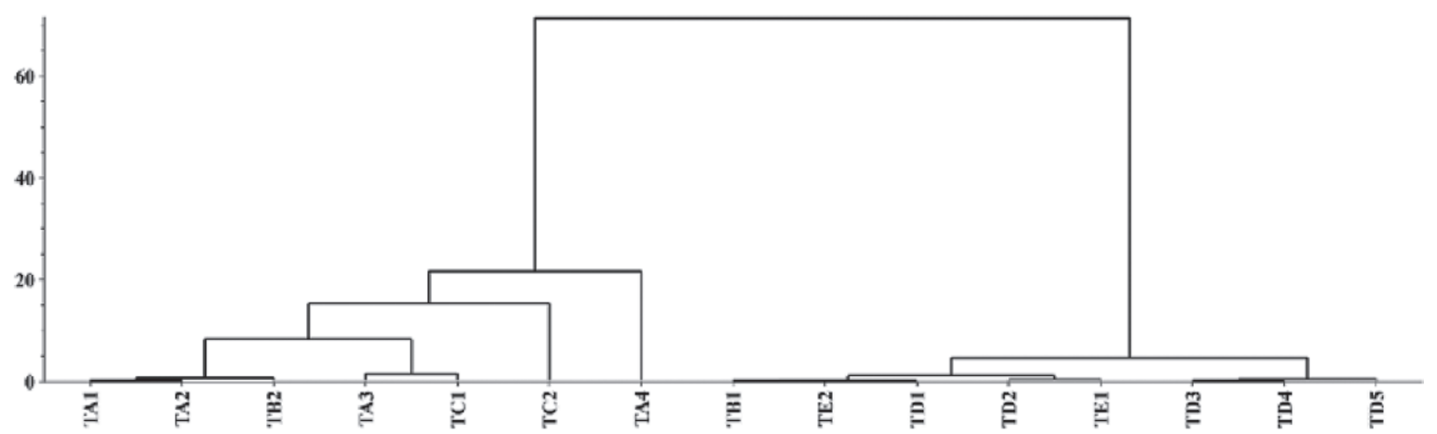

$\mathrm{C}$

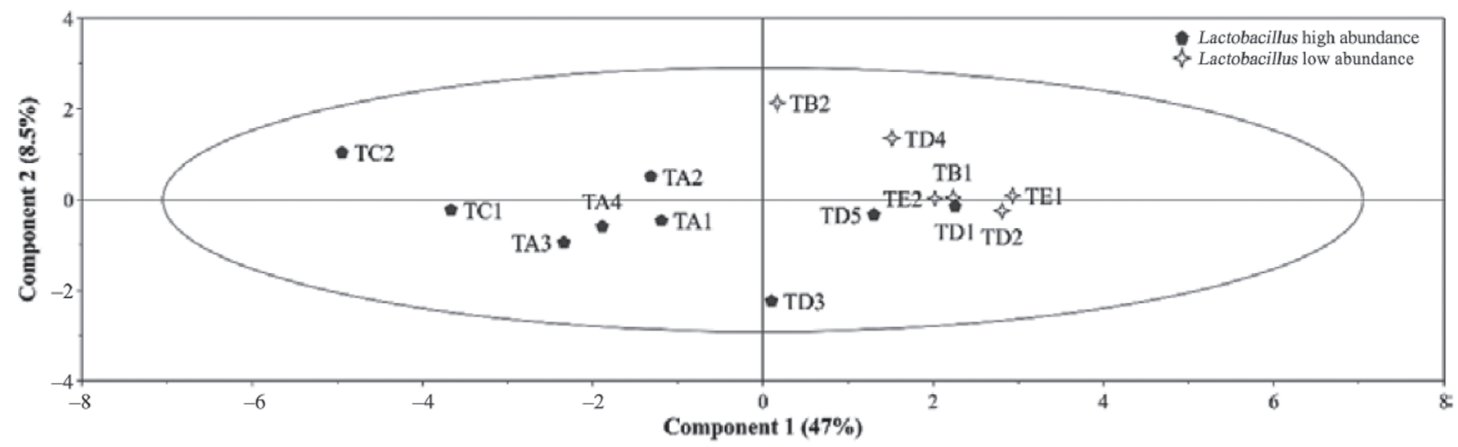

$\mathrm{D}$

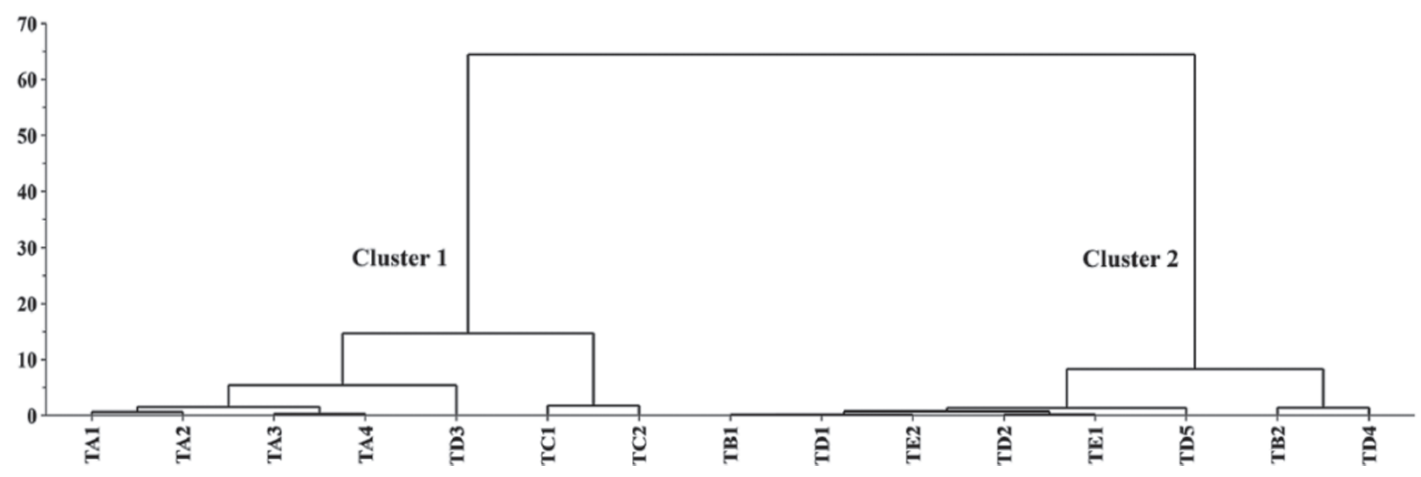

Figure 3. Statistical analysis of volatile flavor compounds in fermented yak milk (T). Each sampling code (TA1-TE2) indicates the individual family who produced the fermented milk; see Table 1 for detailed information. (A) Principal component analysis (PCA); different symbol shapes represent samples from different villages. (B) Hierarchical clustering analysis (HCA), performed according to the scores resulting from PCA. (C) Partial least squares discrimination analysis (PLS-DA). Lactobacillus high (black pentagons) or low (open diamonds) abundance represent samples with relative abundance of Lactobacillus greater or less than $40 \%$ at genus level, respectively. (D) HCA performed according to the scores of PLS-DA. 
and $\mathrm{E}$ (4.32 to $10.39 \mu \mathrm{g} / \mathrm{kg}$ ). The samples from village $\mathrm{C}$ also displayed the highest content of ethanol, ranging from 93.39 to $272.94 \mu \mathrm{g} / \mathrm{kg}$. In general, samples from village $\mathrm{E}$ contained the lowest concentrations of volatile compounds and showed no presence of nonanoic acid, decanoic acid, or ethyl acetate.

The hierarchical clustering analysis of the scores of principle component analysis (Figure 3B) showed that the fermented yak milk samples were divided into 2 groups. The first cluster contained all the samples from villages $\mathrm{A}$ and $\mathrm{C}$; the second cluster, all those from villages $\mathrm{D}$ and $\mathrm{E}$. However, the 2 samples from village $\mathrm{B}$ did not cluster together. It appeared that the volatile profiles of fermented yak milk samples were different among the samples collected from different villages.

Partial least squares discrimination analysis of the volatile compounds in fermented yak milk is shown in Figure 3C, with Lactobacillus abundance differentiating around $40 \%$. According to the variable importance for the projection (VIP), the differential compounds within the volatile profile (VIP $>1$ ) included 2,3-butanedione, hexanoic acid, benzaldehyde, octanoic acid, acetone, disulfide dimethyl, benzoic acid, nonanoic acid, and ethyl acetate. According to the scores of partial least squares discrimination analysis (Figure 3D), the hierarchical clustering analysis indicated that all the samples were divided into 2 clusters. The samples in cluster 2 had lower total content of volatile compounds than those in cluster 1, ranging from 37.8 to $203.96 \mu \mathrm{g} / \mathrm{kg}$ in cluster 2 and 228.18 to $742.32 \mu \mathrm{g} /$ $\mathrm{kg}$ in cluster 1. The Streptococcus-predominant and Lactococcus-predominant samples all grouped in cluster 2. On the other hand, variation was observed for the Lactobacillus-predominant samples, which spread into 2 clusters. Nevertheless, all the samples in cluster 1 were Lactobacillus-predominant in bacterial composition at the genus level. It has been demonstrated that many factors affect the generation of volatile compounds during fermentation, such as microbial composition, raw materials, and fermentation conditions (Cheng, 2010; Settachaimongkon et al., 2014). Thus, further analysis is desired for potential correlation between volatile profile and microbial composition of the fermented yak milk samples.

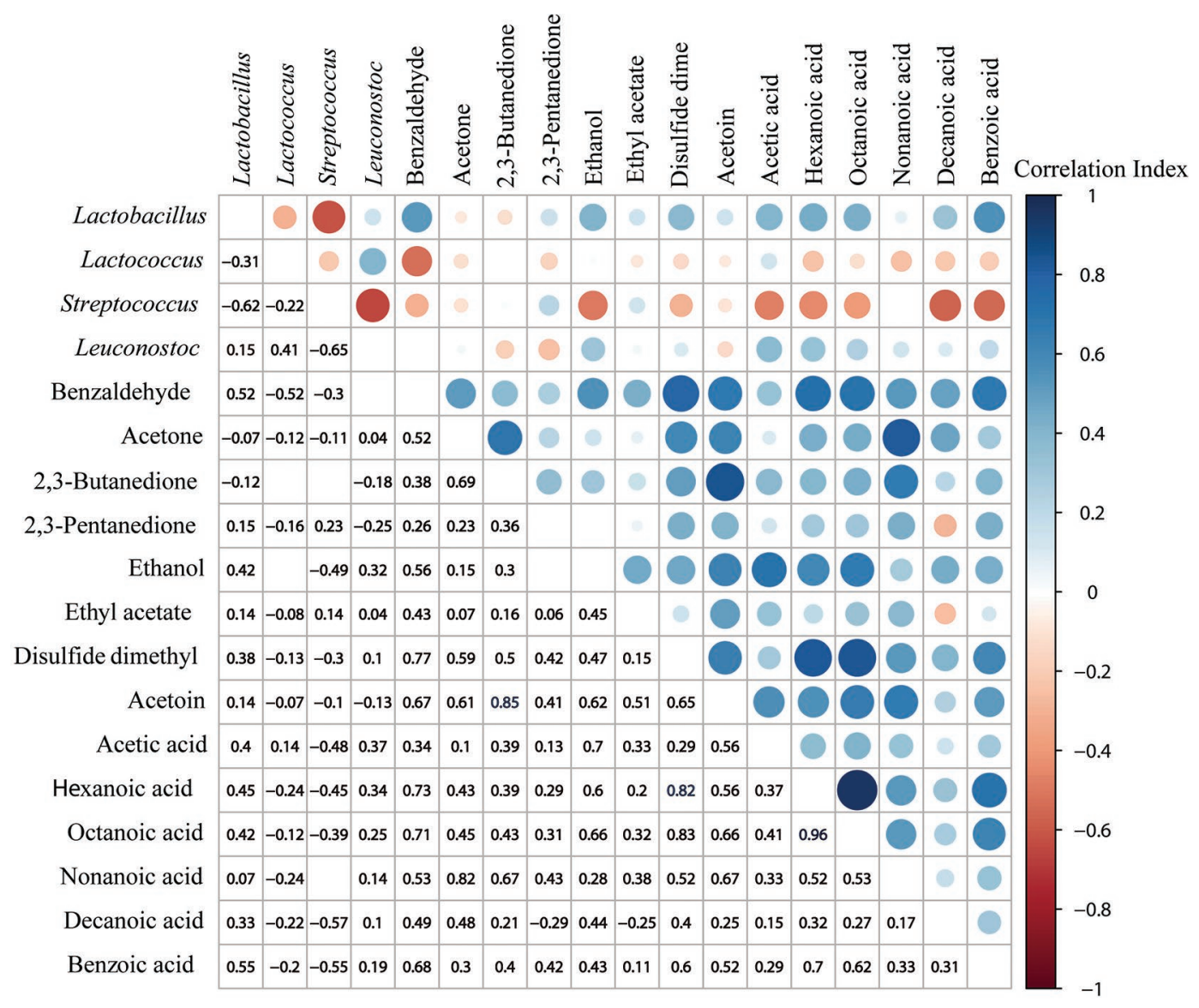

Figure 4. Correlation analysis of lactic acid bacteria genus composition and content of volatile flavor compounds. Pearson coefficients were calculated and considered to be significant when $P<0.05$. Significant correlated relationships are shown in the figure. 


\section{Correlation Analysis Between Volatile Profile and Lactic Acid Bacteria at Genus Level}

The correlation analysis of lactic acid bacteria composition of the fermented yak milk samples indicated a negative correlation between the relative abundance of Lactobacillus with Streptococcus and Lactococcus in the present study (Figure 4). Previous research on crossfeeding interactions in Streptococcus and Lactobacillus showed that the 2 strains could exchange precursors, cofactors, long-chain fatty acids, and other compounds that are associated with biosynthesis or metabolism reactions, and promoted one another's growth (Partanen et al., 2001; Sybesma et al., 2003). However, research has also revealed that the interaction of co-occurring microorganisms is complicated, and cross-feeding interactions can change into competition relationships among microorganisms over time during fermentation. When the proteolytic activity of L. delbrueckii was insufficient to meet the demands for branched-chain amino acids and sulfur amino acids by both Streptococcus thermophilus and L. delbrueckii, competition was observed in the mixed culture (Sieuwerts et al., 2010). The negative correlation between the relative abundance of Lactobacillus and Streptococcus in the fermented yak milk samples seems to indicate competitive behavior between the 2 bacteria after extended fermentation of yak milk for 12 to $36 \mathrm{~h}$, as observed in the collected samples.

Correlations among the bacterial genera of the fermented milk samples and their content of volatile compounds (Figure 4) implies that the relative abundance of Lactobacillus in fermented yak milk had significant positively correlation with the contents of most of the detected volatile compounds, except acetone and butanedione. In contrast, the relative abundance of Streptococcus had significant positive correlation with the content of 2,3-pentanedione and ethyl acetate, and significant negative correlation with the other compounds except 2,3-butanedione. The relative abundance of Lactococcus had significant positive correlation only with the content of acetic acid.

The generation of volatile compounds in fermented milk, including lactic acid, acetaldehyde, diacetyl, acetoin, and ethanol, involves complex biochemical reactions among carbohydrates, proteins, and lipids (Cheng, 2010; De Prisco et al., 2017). The genetic and metabolic diversity of lactic acid bacteria at the genus and strain levels has been well documented and is responsible for flavor diversification in fermented products (Singh et al., 2007). Therefore, thorough characterization of microbiota profiles is required in order to correlate the variations in flavor profiles observed in the present study. We must also point out that a vari- ety of other factors besides lactic acid bacteria might contribute to the volatile profiles of the fermented milk, including autolytic microorganisms, fungi, fermentation conditions, and more.

\section{CONCLUSIONS}

Variation in abundance of bacteria at the genus level was recorded among fermented yak milk samples. Lactobacillus was the most abundant genus in 9 out of 15 fermented yak milk samples. For the rest of the samples, Streptococcus predominated in 4, and Lactococcus predominated in 2 . The volatile profiles of fermented yak milk samples were different among samples collected from different villages. Correlations between bacterial composition and volatile compounds of the traditional fermented yak milk samples were observed. The factors that contributed to the flavor profile of the fermented milk are complicated, and further research needs to be done to reveal the correlation between microbial ecology and flavor profiles of fermented yak milk.

\section{ACKNOWLEDGMENTS}

This work was supported by the National Natural Science Foundation of China (No. 31871829; Beijing), Agricultural Science and Technology Innovation Fund of Jiangsu Province [CX(17)1005; Suzhou, China], National First-Class Discipline Program of Food Science and Technology (JUFSTR20180102), the Fundamental Research Funds for the Central Universities (JUSRP1903B), and Collaborative Innovation Centre of Food Safety and Quality Control in Jiangsu Province (Wuxi, China), the Postgraduate Research and Practice Innovation Program of Jiangsu Province (KYCX19_1818), and the Shanghai Engineering Center of Dairy Biotechnology (19DZ2281400; Shanghai, China). We thank Stephanie Clark from Iowa State University (Ames) for review of the paper for grammar and syntax.

\section{REFERENCES}

Bai, M., M. Qing, Z. Guo, Y. Zhang, X. Chen, Q. Bao, H. Zhang, and T. S. Sun. 2010. Occurrence and dominance of yeast species in naturally fermented milk from the Tibetan Plateau of China. Can. J. Microbiol. 56:707-714. https://doi.org/10.1139/W10-056.

Bao, Q., J. Yu, W. Liu, M. Qing, W. Wang, X. Chen, F. Wang, M. Li, H. Wang, Q. Lv, and H. Zhang. 2012. Predominant lactic acid bacteria in traditional fermented yak milk products in the Sichuan Province of China. Dairy Sci. Technol. 92:309-319. https://doi .org/10.1007/s13594-012-0061-x.

Bergonzelli, G. E., D. Granato, R. D. Pridmore, L. F. Marvin-Guy, D. Donnicola, and I. E. Corthesy-Theulaz. 2006. GroEL of Lactobacillus johnsonii La1 (NCC 533) is cell surface associated: Potential role in interactions with the host and the gastric pathogen Helicobacter pylori. Infect. Immun. 74:425-434. https://doi.org/10.1128/ IAI.74.1.425-434.2006. 
Blaiotta, G., V. Fusco, D. Ercolini, M. Aponte, O. Pepe, and F. Villani. 2008. Lactobacillus strain diversity based on partial hsp60 gene sequences and design of PCR-restriction fragment length polymorphism assays for species identification and differentiation. Appl. Environ. Microbiol. 74:208-215. https://doi.org/10.1128/ AEM.01711-07.

Chen, Y., T. Sun, J. Wang, C. Airden, M. Bai, and H. Zhang. 2009. Comparison of nutrition and microbiological compositions between two types of fermented milk from Tibet in China. Int. J. Food Sci. Nutr. 60 (Suppl. 7):243-250. https://doi.org/10.1080/ 09637480903005540.

Cheng, H. 2010. Volatile flavor compounds in yogurt: A review. Crit. Rev. Food Sci. Nutr. 50:938-950. https://doi.org/10.1080/ 10408390903044081.

Chi, X. L., M. H. Pan, K. Muratzhan, B. G. Sun, B. Wang, and N. S. Ai. 2017. Analysis of volatile flavor compounds of yak milk powder by GC-MS, GC-O-MS combined with sensory evaluation. Sci. Tech. Food Ind. http://www.en.cnki.com.cn/Article_en/ CJFDTotal-SPKJ201717045.htm.

Dan, T., D. Wang, R. L. Jin, H. P. Zhang, T. T. Zhou, and T. S. Sun. 2017. Characterization of volatile compounds in fermented milk using solid-phase microextraction methods coupled with gas chromatography-mass spectrometry. J. Dairy Sci. 100:2488-2500. https://doi.org/10.3168/jds.2016-11528.

De Prisco, A., H. J. F. van Valenberg, V. Fogliano, and G. Mauriello. 2017. Microencapsulated starter culture during yoghurt manufacturing, effect on technological features. Food Bioproc. Tech. 10:1767-1777. https://doi.org/10.1007/s11947-017-1946-8.

Duan, Y., Z. Tan, Y. Wang, Z. Li, Z. Li, G. Qin, Y. Huo, and Y. Cai. 2008. Identification and characterization of lactic acid bacteria isolated from Tibetan qula cheese. J. Gen. Appl. Microbiol. 54:51-60. https://doi.org/10.2323/jgam.54.51.

Fontana, C., D. Bassi, C. López, V. Pisacane, M. C. Otero, E. Puglisi, A. Rebecchi, P. S. Cocconcelli, and G. Vignolo. 2016. Microbial ecology involved in the ripening of naturally fermented llama meat sausages. A focus on lactobacilli diversity. Int. J. Food Microbiol. 236:17-25. https://doi.org/10.1016/j.ijfoodmicro.2016.07.002.

He, S., Y. Ma, J. Wang, Q. Li, X. Yang, S. Tang, and H. Li. 2011. Milk fat chemical composition of yak breeds in China. J. Food Compos. Anal. 24:223-230. https://doi.org/10.1016/j.jfca.2010.07.008.

Hettinga, K. A., H. J. Van Valenberg, T. J. Lam, and A. C. van Hooijdonk. 2008. Detection of mastitis pathogens by analysis of volatile bacterial metabolites. J. Dairy Sci. 91:3834-3839. https://doi.org/ 10.3168/jds.2007-0941.

Hu, L., W. Lu, L. Wang, M. Pan, H. Zhang, J. Zhao, and W. Chen. 2017. Assessment of Bifidobacterium species using groEL Gene on the basis of Illumina MiSeq high-throughput sequencing. Genes (Basel) 8:E336. https://doi.org/10.3390/genes8110336.

Hunt, D. J., H. C. Parkes, and I. D. Lumley. 1997. Identification of the species of origin of raw and cooked meat products using oligonucleotide probes. Food Chem. 60:437-442. https://doi.org/10 .1016/S0308-8146(96)00364-0.

Jia, H. R., L. L. Geng, Y. H. Li, Q. Wang, Q. Y. Diao, T. Zhou, and P. L. Dai. 2016. The effects of Bt Cry1Ie toxin on bacterial diversity in the midgut of Apis mellifera ligustica (Hymenoptera: Apidae). Sci. Rep. 6:24664. https://doi.org/10.1038/srep24664.

Liu, W., X. Xi, Q. Sudu, L. Kwok, Z. Guo, Q. Hou, B. Menhe, T. Sun, and H. Zhang. 2015. High-throughput sequencing reveals microbial community diversity of Tibetan naturally fermented yak milk. Ann. Microbiol. 65:1741-1751. https://doi.org/10.1007/s13213 -014-1013-x.

Liu, W. J., Z. H. Sun, Y. B. Zhang, C. L. Zhang, M. Menghebilige, M. Yang, T. S. Sun, Q. H. Bao, W. Chen, and H. P. Zhang. 2012. A survey of the bacterial composition of kurut from Tibet using a culture-independent approach. J. Dairy Sci. 95:1064-1072. https:/ /doi.org/10.3168/jds.2010-4119.

Mao, B., D. Li, J. Zhao, X. Liu, Z. Gu, Y. Q. Chen, H. Zhang, and W. Chen. 2015. Metagenomic insights into the effects of fructooligosaccharides (FOS) on the composition of fecal microbiota in mice. J. Agric. Food Chem. 63:856-863. https://doi.org/10.1021/ jf505156h.

Milani, C., S. Duranti, M. Mangifesta, G. A. Lugli, F. Turroni, L. Mancabelli, A. Viappiani, R. Anzalone, G. Alessandri, M. C. Ossiprandi, D. van Sinderen, and M. Ventura. 2018. Phylotype-level profiling of lactobacilli in highly complex environments by means of an internal transcribed spacer-based metagenomic approach. Appl. Environ. Microbiol. 84:e00706-e00718. https://doi.org/10 .1128/AEM.00706-18.

Partanen, L., N. Marttinen, and T. Alatossava. 2001. Fats and fatty acids as growth factors for Lactobacillus delbrueckii. Syst. Appl. Microbiol. 24:500-506. https://doi.org/10.1078/0723-2020-00078.

Settachaimongkon, S., M. J. Nout, E. C. Antunes Fernandes, K. A. Hettinga, J. M. Vervoort, T. C. van Hooijdonk, M. H. Zwietering, E. J. Smid, and H. J. van Valenberg. 2014. Influence of different proteolytic strains of Streptococcus thermophilus in co-culture with Lactobacillus delbrueckii ssp. bulgaricus on the metabolite profile of set-yoghurt. Int. J. Food Microbiol. 177:29-36. https://doi.org/ 10.1016/j.ijfoodmicro.2014.02.008.

Shevtsov, A. B., A. R. Kushugulova, I. K. Tynybaeva, S. S. Kozhakhmetov, A. B. Abzhalelov, K. T. Momynaliev, and L. G. Stoyanova 2011. Identification of phenotypically and genotypically related Lactobacillus strains based on nucleotide sequence analysis of the groEL, rpoB $r p l B$, and 16S rRNA genes. Microbiology 80:659-668. https://doi.org/10.1134/S0026261711050134.

Sieuwerts, S., D. Molenaar, S. A. F. T. van Hijum, M. Beerthuyzen, M. J. A. Stevens, P. W. M. Janssen, C. J. Ingham, F. A. M. de Bok, W. M. de Vos, and J. E. T. van Hylckama Vlieg. 2010. Mixed-culture transcriptome analysis reveals the molecular basis of mixed-culture growth in Streptococcus thermophilus and Lactobacillus bulgaricus. Appl. Environ. Microbiol. 76:7775-7784. https: //doi.org/10.1128/AEM.01122-10.

Singh, T. K., K. R. Cadwallader, and M. Drake. 2007. Biochemical processes in the production of flavor in milk and milk products. Pages 715-748 in Handbook of Food Products Manufacturing. Y. H. Hui, ed. John Wiley and Sons Inc., Hoboken, NJ. https://doi .org/10.1002/9780470113554.ch78

Sybesma, W., M. Starrenburg, L. Tijsseling, M. H. N. Hoefnagel, and J. Hugenholtz. 2003. Effects of cultivation conditions on folate production by lactic acid bacteria. Appl. Environ. Microbiol. 69:4542-4548. https://doi.org/10.1128/AEM.69.8.4542-4548.2003.

Xie, M., M. Pan, Y. Jiang, X. Liu, W. Lu, J. Zhao, H. Zhang, and W. Chen. 2019. groEL gene-based phylogenetic analysis of Lactobacillus species by high-throughput sequencing. Genes. 10:530. https:/ /doi.org/10.3390/genes10070530

Yang, M., G. D. Zhang, J. T. Yang, D. Sun, P. C. Wen, and W. B. Zhang. 2018. Effect of $\mathrm{pH}$ on dissociation of casein micelles in yak skim milk. J. Dairy Sci. 101:2998-3007. https://doi.org/10.3168/ jds.2017-13653.

Zhang, H., J. Xu, J. Wang, T. Sun, H. Li, and M. Guo. 2008. A survey on chemical and microbiological composition of kurut, naturally fermented yak milk from Qinghai in China. Food Control 19:578586. https://doi.org/10.1016/j.foodcont.2007.06.010.

\section{ORCIDS}

Xiaoming Liu (1) https://orcid.org/0000-0002-9128-2204

Wei Chen (® https://orcid.org/0000-0003-3348-4710 